Bull. Austral. Math. Soc.

06в $05,08 \mathrm{~B} 05,20 \mathrm{M} 14$

VOL. 48 (1993) [151-162]

\title{
POLYNOMIAL DENSITY OF COMMUTATIVE SEMIGROUPS
}

\section{ANDRZEJ KISIELEWICZ AND NORBERT NEWRLY}

An algebra is said to be polynomially $\boldsymbol{n}$-dense if all equational theories extending the equational theory of the algebra with constants have a relative base consisting of equations in no more than $n$ variables. In this paper, we investigate polynomial density of commutative semigroups. In particular, we prove that, for $n>1$, a commutative semigroup is $(n-1)$-dense if and only if its subsemigroup consisting of all $n$-factor-products is either a monoid or a union of groups of a bounded order. Moreover, a commutative semigroup is $\mathbf{0}$-dense if and only if it is a bounded semilattice. For semilattices, we give a full description of the corresponding lattices of equational theories.

\section{INTRODUCTION}

In [4], considering the general problem of describing the lattices of equational theories of algebras, the authors introduced the concept of well-behaved algebras. They call an algebra well-behaved if every equation (in the language of the algebra with all constants), follows from its constant consequences. Such a property implies that the lattice of equational theories extending the theory of the algebra with constants is isomorphic to the congruence lattice of the algebra. This was used to prove that all infinitely distributive algebraic lattices with compact 1 , as well as algebraic lattices with unique dual atom are representable as lattices of equational theories $[4,7]$.

The property of being well-behaved is equivalence invariant, that is, in fact, the property of the clone of polynomial functions of the algebra, and roughly speaking, it means that there is not much space among polynomials to make identifications.

In [6], the concept was extended to the concept of polynomial density as follows. An equation with constants (a pair of polynomial functions) is $n$-derivable if it follows (in the theory of the algebra with constants) from its $n$-ary consequences. An algebra is (polynomially) $n$-dense, if all equations are $n$-derivable. This is equivalent to saying that equational theories extending the equational theory of the algebra with constants have a relative base consisting of equations in no more than $n$ variables.

In [6], the polynomial density is calculated for some well known classes of algebras. In particular, finite lattices turn out to be 0 -dense (well-behaved), while abelian groups, modules, unary algebras and commutative monoids are 1-dense.

Received 17th September, 1992

Copyright Clearance Centre, Inc. Serial-fee code: 0004-9729/93 \$A2.00+0.00. 
In this paper we examine the density of commutative semigroups. One aim is to provide examples and intuitions for further more general studies. Yet, polynomial density for semigroups seems to be of an independent interest, because theories involving constants are closely connected with presentations of semigroups, a classical topic in semigroup theory.

In Section 1, we deal with semilattices. From [4] it follows that bounded semilattices are 0 -dense. We show that, in general, semilattices are 1-dense. In this special case, it is possible, in fact, to describe the whole lattice of equational theories extending the theory of a semilattice with constants. If the semilattice does not happen to be 0 -dense, then this lattice is no longer isomorphic to the congruence lattice. Yet, our description shows, in particular, that it is isomorphic to the congruence lattice of the semilattice obtained from the original one by adjoining an identity and a zero in a natural way.

Next we turn to characterising the property of being polynomially $n$-dense for all commutative semigroups. The case $n=0$ turned out to require a separate treatment. In Section 2, we show that a commutative semigroup is 0 -dense (well-behaved) if and only if it is a bounded semilattice. In Section 3 the remaining cases are handled. In particular, we show that for $n>1$ a commutative semigroup $S$ is $(n-1)$-dense if and only if its subsemigroup $\mathbf{S}^{(n)}$ of $n$-factor-products is either a monoid or a union of groups of a bounded order.

We assume that the reader is familiar with basic concepts of semigroup theory, lattice theory and universal algebra.

Throughout the paper, by $C$ on $(\mathbf{A})$ we denote the lattice of congruence relations of an algebra $\mathbf{A}$, and by $\operatorname{Eqc}(\mathbf{A})$, the lattice of equational theories extending the theory of $\mathbf{A}$ with constants (that is, the type of $\mathbf{A}$ is expanded with nullary operations for all elements of $A$ ). In a semigroup, the zero element and the identity element are denoted by 0 and 1 , respectively.

\section{Semilattices}

Semilattices are idempotent commutative semigroups, and every semilattice term is of the form $u=x_{1} \ldots x_{n}$. Hence, every equation in the language of the semilattice with constants is equivalent to one of the form
(a) $u=v$,
(b) $u a=v$,
(c) $u a=u b$,

where $u, v$ are semilattice terms, $a, b$ are constants, and $u, v$ are allowed to be empty words, if followed by a constant.

Assume that $u$ and $v$ are distinct, that is, there is a variable occurring only in one of them. Then (using idempotency), from $u=v$ one can derive both $x=x a$ and 
$a=x a$, and in consequence, $x=a$. Conversely, from $x=a$ one can derive $x=y$, and therefore, $u=v$. This means, that in this case, any $u=v$ is equivalent to $x=a$, where $a$ is an arbitrary constant.

Similarly, $u a=v$ is equivalent to $x=a$, and $u a=v b$ can be easily shown to be equivalent to the pair of equations: $a=b$ and $x a=a$.

If $u$ and $v$ are the same, then equation (a) belongs to every theory, while $u a=u$ is equivalent to $x a=x$; and $u a=u b$ implies $a=a b$ and $b a=b$, and therefore is equivalent to $a=b$.

Summarising we have the following

Proposition 1. Every equational theory extending the equational theory $\boldsymbol{\Sigma}_{\mathbf{0}}$ of a semilattice with constants is determined by the set of equations $\Sigma_{0} \cup \Sigma$, where $\Sigma$ contains only equations of the form $a=b, x=x a$, and $x a=a$.

In particular, every semilattice is polynomially 1-dense.

If the semilattice $S$ has 1 , then an equation of the form $x=x a$ is equivalent to the equation $a=1$; if the semilattice has 0 , then an equation of the form $x a=a$ is equivalent to the equation $a=0$. In consequence, we have

COROLlaRY 2. Every bounded semilattice is polynomially 0-dense.

We turn to describing the lattice of equational theories involved here.

For an equational theory $\Sigma$ of a semilattice $S$ let $\psi_{\Sigma}$ be the set of all pairs $(a, b)$ for which the equation $a=b$ is in $\Sigma, F_{\Sigma}$, the set of all elements $a$ of $S$ for which the equation $x=x a$ is in $\Sigma$, and $I_{\Sigma}$, the set of all elements $a$ of $S$ for which the equation $x a=a$ is in $\Sigma$. Clearly $\psi_{\Sigma}$ is a congruence relation of $\mathbf{S}, F_{\Sigma}$ is a (meet-semilattice) filter in $\mathbf{S}$ and $I_{\Sigma}$ is an (order) ideal in $\mathbf{S}$. Moreover, each triple $(\psi, F, I)=\left(\psi_{\Sigma}, F_{\Sigma}, I_{\Sigma}\right)$ satisfies the following conditions:

(F1) For all $a \in F$ and for all $c \in \mathrm{S}$, the pair $(c, a c)$ is a member of $\psi$.

(F2) If $(a, b) \in \psi$ and $a \in F$, then $b \in F$.

(I1) For all $a \in I$ and for all $c \in \mathrm{S}$, the pair $(a c, a)$ is a member of $\psi$.

(I2) If $(a, b) \in \psi$ and $a \in I$, then $b \in I$.

We need a concept known as Day's doubling construction. An upper (lower) pseudointerval $P$ of a lattice $L$ is the union of intervals having a common greatest (least) element. For such a pseudointerval $P$ the lattice $L[P]$ is defined by replacing $P$ in $L$ by the product $P \times \mathbf{2}$ of $P$ and the two-element chain; the order is extended in a natural way. For a complete description see [1].

Now, given two order filters $P_{1}$ and $P_{2}$ of a lattice $L$ we construct $L\left[P_{1}\right]$. Then, the union of $P_{2}$ and the upper copy of $P_{1} \cap P_{2}$ forms an order filter in this lattice. Doubling this we get a new lattice, which we denote by $L\left[P_{1}, P_{2}\right]$.

For a groupoid A let $C o n_{1}(\mathrm{~A})$ be the set of all congruences of $\mathrm{A}$ such that the 
factor groupoid has an identity. Let $C_{0 n_{0}}(\mathbf{A})$ be the set of all congruences of $\mathbf{A}$ such that the factor groupoid has a zero. Both $C o n_{1}(\mathbf{A})$ and $C o n_{0}(\mathbf{A})$ are order filters, and therefore upper pseudointervals of $C$ on $(\mathbf{A})$. For a groupoid $\mathbf{A}$ let $\mathbf{A}+1$ be the groupoid with an additional identity element, and $\mathbf{A}+0$, the groupoid with an additional zero element.

Lemma 3. For every groupoid $\mathbf{A}$, the congruence lattice of $\mathbf{A}+\mathbf{1}$ is isomorphic to $\operatorname{Con}(\mathbf{A})\left[\operatorname{Con}_{1}(\mathbf{A})\right]$, and the congruence lattice of $\mathbf{A}+0$ is isomorphic to $\operatorname{Con}(\mathrm{A})\left[\operatorname{Con}_{0}(\mathrm{~A})\right]$.

Proof: Every congruence of $\mathbf{A}$ can be made to a congruence of $\mathbf{A}+1$ by adding a block containing 1 alone. Let $\psi$ be a congruence of $\mathbf{A}+1$ where 1 is not alone in its block. Then the restriction of $\psi$ to $A, \psi \cap A^{2}$, is a congruence of $\mathbf{A}$, and the corresponding factor groupoid has an identity, namely the class of those $a$ 's with $a \psi 1$. For every such $a$ and any $b$ the elements $a b, b a$ and $b$ are congruent modulo $\psi \cap A^{2}$. Hence, $\psi \cap A^{2}$ is contained in $\operatorname{Con}_{1}(\mathbf{A})$. The restriction of congruences of $\mathbf{A}+1$ to $\mathbf{A}$ is a surjective lattice homomorphism under which every congruence of $\mathbf{A}$ has at most two preimages, and it has two preimages if and only if it is contained in $\operatorname{Con}_{1}(\mathbf{A})$. This establishes an isomorphism between $\operatorname{Con}(\mathbf{A}+1)$ and $\operatorname{Con}(\mathbf{A})\left[\operatorname{Con}_{1}(\mathbf{A})\right]$. The proof of the second statement is similar.

For a semilattice $\mathbf{S}$, by $\mathbf{S}_{0}^{1}$ we denote the semilattice obtained by adding 0 and 1 , whenever either of them is missing. Define $L^{*}\left[P_{1}, P_{2}\right]$ for two order filters $P_{1}$ and $P_{2}$ of a lattice $L$ to be $L\left[P_{1}, P_{2}\right]$, if both $P_{1}$ and $P_{2}$ are not the whole lattice $L$, and $L\left[\emptyset, P_{2}\right]=L\left[P_{2}\right], L\left[P_{1}, \emptyset\right]=L\left[P_{1}\right]$, or $L[\emptyset, \emptyset]=L$ if, respectively, $P_{1}, P_{2}$, or both are equal to $L$. Then we have

THEOREM 4. For a semilattice $S$ the following lattices are isomorphic:

(i) $\operatorname{Eqc}(\mathrm{S})$,

(ii) $\operatorname{Con}(\mathrm{S})^{*}\left[\operatorname{Con}_{0}(\mathrm{~S}), \operatorname{Con}_{1}(\mathrm{~S})\right]$,

(iii) $\operatorname{Con}\left(\mathbf{S}_{0}^{1}\right)$.

ProOF: In view of Proposition 1 every equational theory $\Sigma$ in $E q c(S)$ is uniquely determined by its triple $\left(\psi_{\Sigma}, I_{\Sigma}, F_{\Sigma}\right)$. On the other hand, every triple $(\psi, I, F)$ satisfying (F1), (F2), (I1) and (I2) gives rise to such a theory.

If $I_{\Sigma}$ is not empty, then $I_{\Sigma}$ is a block of the congruence $\psi_{\Sigma}$ because of condition (I2), and it is the least block because of condition (I1). Hence, if $I_{\Sigma}$ is not empty, then $\psi_{\Sigma}$ belongs to $C o n_{0}(S)$ and $I_{\Sigma}$ is uniquely determined by $\psi_{\Sigma}$. If $F_{\Sigma}$ is not empty, then $F_{\Sigma}$ is a block of the congruence $\psi_{\Sigma}$ because of condition (F2), and it is the greatest block because of condition (F1). Hence, if $F_{\Sigma}$ is not empty, then $\psi_{\Sigma}$ belongs to $C o n_{1}(\mathrm{~S})$ and $F_{\Sigma}$ is uniquely determined by the congruence $\psi_{\Sigma}$. If the semilattice $\mathbf{S}$ has 0 , then it is always contained in $I_{\Sigma}$, and $\operatorname{Con}_{0}(\mathbf{S})=\operatorname{Con}(\mathbf{S})$. If $\mathbf{S}$ has 1 , then 
it is always contained in $F_{\Sigma}$, and $C o n_{1}(\mathrm{~S})=\operatorname{Con}(\mathrm{S})$.

Therefore, in view of Lemma 3, the isomorphism between the lattices $E q c(S)$ and $\operatorname{Con}(\mathbf{S})^{*}\left[\operatorname{Con}_{0}(\mathbf{S}), \operatorname{Con}_{1}(\mathrm{~S})\right]$ can be defined in the following way. If $\mathbf{S}$ has no 0 and no 1 , than an equational theory $\Sigma$ is mapped upon the congruence $\psi_{\Sigma}$, whenever $F_{\Sigma}$ and $I_{\Sigma}$ are empty, or upon the corresponding copy of $\psi_{\Sigma}$, whenever $F_{\Sigma}$ or $I_{\Sigma}$ or both are not empty (for example, if $F_{\Sigma}$ is empty, and $I_{\Sigma}$ not, then it is mapped on the copy of $\psi_{\Sigma}$ in the upper level of the doubled filter $\operatorname{Con}_{1}(\mathbf{S})$, et cetera).

If $\mathbf{S}$ has 0 , but no 1 , then $\operatorname{Con}(\mathbf{S})^{*}\left[\operatorname{Con}_{0}(\mathbf{S}), \operatorname{Con}_{1}(\mathbf{S})\right]=\operatorname{Con}(\mathbf{S})\left[\operatorname{Con}_{1}(\mathbf{S})\right]$ and the isomorphism is defined analogously, according to whether $F_{\Sigma}$ is or is not empty. The case with 1, but no 0 is similar. If there are both 0 and 1 in $\mathbf{S}$, then $\operatorname{Eqc}(\mathbf{S})$ is isomorphic to $\operatorname{Con}(\mathrm{S})$.

The results above can be interpreted in the following way: If there are 1 and 0 in a semilattice, all equations follow from their constant consequences. If 1 or 0 are missing, then equations $a x=x$ or $a x=a$ are substitutes for the constant equations $a=1$ or $a=0$. No other equations are needed to describe the lattice of equational theories.

\section{Characterisation of 0-Dense commutative semigroups}

For two unary terms $f, g$ in the language of a semigroup $\mathrm{S}$ with constants, $R(f, g)$ denotes the congruence of $S$ generated by all pairs $(f(a), g(a))$ with $a$ running over the elements of $\mathbf{S}$ (see [3]). Then we have

LemMa 5. In a commutative semigroup $\mathrm{S}$, a unary equation $f=g$ is 0 -derivable if and only if either $f=g$ holds, or there are pairs $\left(a_{i}, b_{i}\right) \in R(f, g)$ and integers $n_{i}$ $(i=0, \ldots, k)$, such that the equations $f=x^{n_{0}} a_{0}, x^{n_{i}} b_{i}=x^{n_{i+1}} a_{i+1}(i=0, \ldots, k-1)$, and $g=x^{n_{k}} b_{k}$ hold.

Proof: It is not hard to see that an equation $s=t$ is 0 -derivable if and only if there are terms $p_{i}$ and $p_{i}^{\prime}$ in the language of an algebra $A$ with constants, $i=$ $0, \ldots, k$, such that $s=p_{0}, p_{i}^{\prime}=p_{i+1}(i=0, \ldots, k-1)$, and $t=p_{k}^{\prime}$ hold in $A$, with $p_{i}=t_{i}\left(x_{1}, \ldots, x_{n}, c_{1}, \ldots, c_{m}\right), p_{i}^{\prime}=t_{i}\left(x_{1}, \ldots, x_{n}, d_{1}, \ldots, d_{m}\right)$, for some term $t_{i}$, and $c_{j}$ congruent to $d_{j}$ modulo $R(s, t)$ (see $[4,3]$, or $[6]$ ). Restricting to unary equations and commutative semigroups yields the desired result.

Using this, we prove the following

THEOREM 6. A commutative semigroup is 0-dense if and only if it is a bounded semilattice.

In view of Corollary 2 we only need to prove that if a commutative semigroup $\mathbf{S}$ is 0 -dense, then it is a semilattice with 0 and 1 . To this end it is enough to consider just two equations. 
LEMmA 7. If an equation $x=a$ is 0 -derivable, then $\mathbf{S}$ is a monoid with 0 .

Proof: In view of Lemma 5 there are positive integers $k, n_{i}$ and $a_{i}, b_{i} \in \mathbf{S}$, $i=0, \ldots, k$, such that $x=x^{n_{0}} a_{0}, x^{n_{i}} b_{i}=x^{n_{i+1}} a_{i+1}, i=0, \ldots, k-1$, and $x^{n_{k}} b_{k}=a$. From the first equation we can conclude that $x a_{0}^{n_{0}}=x^{n_{0}} a_{0}^{n_{0}+1}=x^{n_{0}} a_{0}=x$, so $a_{0}^{n_{0}}=1$ is an identity. Next, by substituting 1 for $x$ we can conclude that $a_{0}=1$, $b_{i}=a_{i+1}$ for $i=0, \ldots, k$, where $a_{k+1}=a$. Define $d$ to be $a_{1} \ldots a_{k+1}$. Then $x d=x a_{1} \ldots a_{k+1}=x^{n_{0}} a_{1} \ldots a_{k+1}=x^{n_{1}} a_{2} \ldots a_{k+1} a_{1}=x^{n_{2}} a_{3} \ldots a_{k+1} a_{1} a_{2}=\ldots=$ $x^{n_{k}} a_{k+1} a_{1} \ldots a_{k}=a_{1} \ldots a_{k+1}=d$. Consequently, $d$ is a zero.

LEMMA 8. If $\mathrm{S}$ is a commutative monoid and the equation $x=x^{2}$ is 0 -derivable, then it holds, that is, $\mathrm{S}$ is a semilattice.

ProOF: As previously, using Lemma 5 and the existence of 1 , we see that there are positive integers $k, n_{i}$ and elements $a_{i}$ congruent to 1 modulo $R\left(x, x^{2}\right), i=0, \ldots, k$, such that $x=x^{n_{0}}, x^{n_{i-1}} a_{i}=x^{n_{i}} a_{i}$ for $i=1, \ldots, k$ and $x^{n_{k}}=x^{2}$. Define $d$ to be $a_{1} \ldots a_{k}$. Then, $x d=x a_{1} \ldots a_{k}=x^{n_{0}} a_{1} \ldots a_{k}=x^{n_{1}} a_{2} \ldots a_{k} a_{1}=x^{n_{2}} a_{3} \ldots a_{k} a_{1} a_{2}=$ $\ldots=x^{n_{k}} a_{1} \ldots a_{k}=x^{2} a_{1} \ldots a_{k}=x^{2} d$. Hence, $x d=x^{2} d$, and $d$ is also congruent to 1 modulo $R\left(x, x^{2}\right)$. What we wish to show is that $d=1$.

From Malcev's description of congruence relations (see [2]), a pair $(c, d)$ of elements of $A$ is congruent modulo $R\left(x, x^{2}\right)$ if and only if there exist elements $a_{i}$ in $A$ and unary polynomial functions $p_{i}$ of $A, i=0, \ldots, k$ such that $c \in\left\{p_{0}\left(a_{0}\right), p_{0}\left(a_{0}^{2}\right)\right\}$, $\left\{p_{i-1}\left(a_{i-1}\right), p_{i-1}\left(a_{i-1}^{2}\right)\right\} \cap\left\{p_{i}\left(a_{i}\right), p_{i}\left(a_{i}^{2}\right)\right\} \neq \emptyset$, and $d \in\left\{p_{k}\left(a_{k}\right), p_{k}\left(a_{k}^{2}\right)\right\}$. Since every unary polynomial function $p_{i}$ can be written in the form $p_{i}(x)=x^{n_{i}} b_{i}$, we can replace $a_{i}$ by $a_{i}^{n_{i}}$ and assume that every $p_{i}$ is the multiplication by a constant $b_{i}$. Hence, $d$ is congruent to 1 modulo $R\left(x, x^{2}\right)$ iff there exist elements $a_{i}$ and $b_{i}$ in $S$, such that $1 \in\left\{a_{0} b_{0}, a_{0}^{2} b_{0}\right\},\left\{a_{i-1} b_{i-1}, a_{i-1}^{2} b_{i-1}\right\} \cap\left\{a_{i} b_{i}, a_{i}^{2} b_{i}\right\} \neq \emptyset$ for $i=1, \ldots, k$ and $d \in\left\{a_{k} b_{k}, a_{k}^{2} b_{k}\right\}$. From the first statement we get that both $a_{0}$ and $b_{0}$ are units (that is, divisors of 1 ). Since a product $a b$ is a unit if and only if both factors $a$ and $b$ are units, it follows by simple induction that all $a_{i}$ and $b_{i}$, and in consequence, also $d$, are units.

It follows that there is an element $a$ such that $a d=1$. But $a=a a d=a^{2} d=a d=$ 1 , since $x d=x^{2} d$, so $d$ is the identity, as required.

The above lemmas prove the "only if" part of the theorem.

\section{Characterisation of $n$-Dense COMmutative semigroups for $n \geqslant 1$}

In this section, we characterise the class of all polynomially $n$-dense commutative semigroups for $n \geqslant 1$. We begin with a necessary condition, which will turn out to be also sufficient. A term $t$ (in the expanded language of a semigroup $S$ ) is called uniquely representable in $\mathrm{S}$, if all the equations $t=u$ belonging to the equational theory of $\mathrm{S}$ 
with constants are trivial (that is, $s$ is equal to $t$ up to associativity and commutativity). In particular, there are no uniquely representable terms in monoids. Note also that, if $t$ is uniquely representable, then every subterm of $t$ is uniquely representable.

LEMMA 9. If the term $x_{1} \ldots x_{n}$ with $n \geqslant 2$ is uniquely representable in $S$, then $\mathrm{S}$ is not $(n-1)$-dense.

Proof: Let $\psi$ be the equivalence relation on the set of terms which identifies all terms but those of the form $x_{i_{1}} \ldots x_{i_{k}}$ with $k \leqslant n$. Since $x_{1} \ldots x_{n}$ and all its subterms are uniquely representable, $\psi$ is a fully invariant congruence relation of the term algebra (with constants). The same is true for $\psi^{\prime} \supseteq \psi$, in which also all terms $x_{i_{1}} \ldots x_{i_{k}}$ with $k=n$ are identified with the constants. Clearly, the equation $x_{1} \ldots x_{n}=c$ is in $\psi^{\prime}$, for any constant $c$. Moreover, all $(n-1)$-ary consequences of this equation are in $\psi$, since the difference between $\psi$ and $\psi^{\prime}$ consists only of equations containing more than $n-1$ variables. But $x_{1} \ldots x_{n}=c$ is not in $\psi$, which shows that $\mathbf{S}$ is not $(n-1)$-dense.

Given a semigroup $S$, let $S^{(n)}$ denote the subsemigroup of those elements $a \in \mathbf{S}$ for which there are $n$ elements $a_{1}, \ldots, a_{n}$ such that $a=a_{1} \ldots a_{n}$. We show how $\mathbf{S}^{(n)}$ is related to the polynomial density of the semigroup $\mathbf{S}$.

Lemma 10. For $n \geqslant 2$, if $\mathbf{S}^{(n)}$ is a monoid, then $S$ is polynomially $(n-1)$-dense.

Proof: Assume that $S$ is not $(n-1)$-dense, and choose $f$ and $g$ such that $f=g$ is not $(n-1)$-derivable and the number of variables occuring in $f$ or $g$ is minimal with respect to this property. Let $a$ be the identity in $S^{(n)}$.

Assume first, that the equation $f=g$ is regular, that is, $f$ and $g$ have the same set of variables. Then both $f$ and $g$ have at least $n$ factors, and therefore the equations $f=f a$ and $g=g a$ hold in $\mathbf{S}$. Hence $f$ can be written as $\Pi x_{i}^{\alpha_{i}} c$ and $g$ can be written as $\Pi x_{i}^{\alpha_{i}^{\prime}} d$, where $c$ and $d$ are constants.

Substituting suitably $a$ in $f=g$, we get as consequences the equations $x_{i}^{\alpha_{i}} c a=$ $x_{i}^{\alpha_{i}^{\prime}} d a$ for all $i$, and $c a=d a$ (since $a$ is an idempotent).

Conversely, from these we can conclude

$$
f=x_{1}^{\alpha_{1}} \ldots x_{k}^{\alpha_{k}} c a=x_{1}^{\alpha_{1}^{\prime}} \ldots x_{k}^{\alpha_{k}^{\prime}} d a=g .
$$

Hence, $f=g$ is in fact 1-derivable, which is a contradiction.

Let $f=g$ be not regular. Then, we may assume that there is a variable $x$ occuring in $f$ but not in $g$, and $f$ is of the form $x^{\alpha} f^{\prime}$ for some term $f^{\prime}$ not containing $x$. The equation $f=g$ clearly is equivalent to the pair of equations $a f^{\prime}=g$ and $a f^{\prime}=f$. But $a f^{\prime}=g$ has less variables than $f=g$, and is therefore $(n-1)$-derivable. Hence the equation $a f^{\prime}=f$ is not $(n-1)$-derivable.

We show that this leads to a contradiction. First, $f$ must contain more than $(n-1)$ variables, and therefore the equation $f=f a$ holds in $\mathrm{S}$. It follows that the 
equation $a f^{\prime}=f$ can be written in the form

$$
x_{1}^{\alpha_{1}} \ldots x_{k}^{\alpha_{k}} c a=x^{\alpha} x_{1}^{\alpha_{1}} \ldots x_{k}^{\alpha_{k}} c a,
$$

for some constant $c$. However, this equation is equivalent to the equation $c a=x^{\alpha} c a$, and hence it is 1-derivable, a contradiction.

In the next lemma we give a sufficient condition for $n$-density in the form of an equation. We shall see later how it is also connected to the subsemigroup $\mathbf{S}^{(n)}$.

LEMMA 11. If the equation

$$
x_{1} \ldots x_{n}=x_{1}^{r+1} \ldots x_{n}
$$

holds in a commutative semigroup $S$ for some $r \geqslant 1$ and $n \geqslant 2$, then $S$ is polynomially $(n-1)$-dense.

Proof: As before, assume that that $f=g$ is not $(n-1)$-derivable and is minimal with respect to the number of variables.

Suppose that $f=g$ is regular, that is, equivalent to an equation of the form

$$
\Pi x_{i}^{\alpha_{1}} a=\Pi x_{i}^{\alpha_{i}^{\prime}} b,
$$

where $i$ runs from 1 to some $k \geqslant n, \alpha_{i}, \alpha_{i}^{\prime}>0$, and $a$ and $b$ are constants, perhaps one or both missing. By replacing all variables with the exception of $x_{i}$ by $x_{i}^{r}$, we get the unary consequences

$$
x_{i}^{\alpha_{i}+c_{i} r} a=x_{i}^{\alpha_{i}^{\prime}+c_{i}^{\prime} r} b
$$

where $c_{i}=\sum_{j \neq i} \alpha_{j}$ and $c_{i}^{\prime}=\sum_{j \neq i} \alpha_{j}^{\prime}$. On the other hand, replacing all variables by $x^{r}$, we derive an equation

$$
x^{c r} a=x^{c^{\prime} r_{b}}
$$

for some positive integers $c$ and $c^{\prime}$. By repeated use of these (and (1)) we can conclude back

$$
f=\Pi x_{i}^{\alpha_{i}} a=\Pi x_{i}^{\alpha_{i}+c_{i} r} a=\cdots=\Pi x_{i}^{\alpha_{i}^{\prime}+c_{i}^{\prime} r} b=g .
$$

Hence $f=g$ is again 1-derivable, a contradiction.

Now suppose that $f=g$ is not regular. As in the proof of the previous lemma we can assume the equation is of the form $f=a f^{\prime}$, where $f=x^{\alpha} f^{\prime}$ for some $f^{\prime}$ not containing $x$ and some constant $a$.

At first, assume also that $f^{\prime}$ does not contain a constant. Then $f=a f^{\prime}$ is of the form

$$
x^{\alpha} x_{1}^{\alpha_{1}} \ldots x_{k}^{\alpha_{k}}=a x_{1}^{\alpha_{1}} \ldots x_{k}^{\alpha_{k}}
$$


with $k \geqslant n-1$, so that (1) can be applied.

Replacing all variables except for $x$ by $a^{r}$, we derive the unary consequence

$$
x^{\alpha} a^{c r}=a a^{c r}
$$

for some $c>0$, while replacing all variables by $x^{r}$, we derive

$$
x^{c^{\prime} r}=a x^{c^{\prime \prime} r}
$$

for some $c^{\prime}, c^{\prime \prime}>0$. From these we can conclude

$$
\begin{aligned}
f & =x^{\alpha} x_{1}^{\alpha_{1}} \ldots x_{k}^{\alpha_{k}}=x^{\alpha^{c^{\prime} r}} x^{\alpha_{1}} \ldots x_{k}^{\alpha_{k}} \\
& =x^{\alpha} a x^{c^{\prime \prime} r} x_{1}^{\alpha_{1}} \ldots x_{k}^{\alpha_{k}}=x^{\alpha^{c r}} a x^{c^{\prime \prime} r} x_{1}^{\alpha_{1}} \ldots x_{k}^{\alpha_{k}} \\
& =x^{\alpha} a^{c r} x^{c^{\prime} r} x_{1}^{\alpha_{1}} \ldots x_{k}^{\alpha_{k}}=a a^{c r} x_{1}^{\alpha_{1}} \ldots x_{k}^{\alpha_{k}}=g .
\end{aligned}
$$

Hence $f=g$ is 1 -derivable, a contradiction.

There remains the case when $f^{\prime}$ contains a constant, that is, $f=a f^{\prime}$ is equivalent to an equation of the form

$$
x^{\alpha} x_{1}^{\alpha_{1}} \ldots x_{k}^{\alpha_{k}} b=a x_{1}^{\alpha_{1}} \ldots x_{k}^{\alpha_{k}} b
$$

Then we can replace all variables with the exception of $x$ by $b$ and obtain

$$
x^{\alpha} b^{c r} b=a b^{c r} b
$$

for some $c$. From this and equation (1) we can get back

$$
f=x^{\alpha} b x_{1}^{\alpha_{1}} \ldots x_{k}^{\alpha_{k}}=x^{\alpha} b^{c r} b x_{1}^{\alpha_{1}} \ldots x_{k}^{\alpha_{k}}=a b^{c r} b x_{1}^{\alpha_{1}} \ldots x_{k}^{\alpha_{k}}=g
$$

which completes the proof.

LEMмA 12. Let $\mathbf{S}$ be a commutative semigroup. If there is an element $a \in \mathbf{S}$ and positive integers $\alpha_{i}$ with $\sum_{i=1}^{n} \alpha_{i}>n$ such that the equation

$$
x_{1} \ldots x_{n}=x_{1}^{\alpha_{1}} \ldots x_{n}^{\alpha_{n}} a
$$

holds in $\mathrm{S}$, then the following equivalent conditions hold:

(i) An equation

$$
x_{1} \ldots x_{n}=x_{1}^{\alpha_{1}} \ldots x_{n}^{\alpha_{n}}
$$

holds in $\mathbf{S}$ for positive integers $\alpha_{i}$ with $\sum_{i=1}^{n} \alpha_{i}>n$. 
(ii) There is an integer $r \geqslant 1$ such that the equation

$$
x_{1} \ldots x_{n}=\left(x_{1} \ldots x_{n}\right)^{r+1}
$$

holds in $\mathbf{S}$.

(iii) There is an integer $r \geqslant 1$ such that the equation

$$
x_{1} \ldots x_{n}=x_{1}^{r+1} x_{2} \ldots x_{n}
$$

holds in $\mathbf{S}$.

Proof: We prove (i). From the equation in the assumption we can conclude

$$
\Pi x_{i}=\Pi x_{i} \Pi x_{i}^{k\left(\alpha_{i}-1\right)} a^{k}
$$

for all integers $k \geqslant 0$. Also we can conclude

$$
a^{n}=a^{\bullet+1}
$$

where $s=\sum \alpha_{i}$. Therefore we have

$$
\begin{aligned}
\Pi x_{i} & =\Pi x_{i} \Pi x_{i}^{(s+1)\left(\alpha_{i}-1\right)} a^{s+1} \\
& =\Pi x_{i} \Pi x_{i}^{n\left(\alpha_{i}-1\right)} a^{n} \Pi x_{i}^{(s+1-n)\left(\alpha_{i}-1\right)} \\
& =\Pi x_{i} \Pi x_{i}^{(s+1-n)\left(\alpha_{1}-1\right)}=\Pi x_{i}^{(s+1-n)\left(\alpha_{i}-1\right)+1} .
\end{aligned}
$$

(i) $\Rightarrow$ (ii) From the equation in (i) we can deduce the equation

$$
\Pi x_{i}=\Pi x_{i} \Pi x_{i}^{\alpha_{g(i)}-1}
$$

where $g$ is any permutation on the set $\{1, \ldots, n\}$. Therefore we have

$$
\begin{aligned}
\Pi x_{i} & =\Pi x_{i} \Pi x_{i}^{\alpha_{i}-1}=\Pi x_{i} \Pi x_{i}^{\alpha_{i+1}-1} \Pi x_{i}^{\alpha_{i}-1} \\
& =\Pi x_{i} \Pi x_{i}^{\alpha_{i+2}-1} \Pi x_{i}^{\alpha_{i+1}-1} \Pi x_{i}^{\alpha_{i}-1} \\
& =\ldots=\Pi x_{i} \Pi x_{i}^{(s-n)}=\Pi x_{i}^{(s-n+1)}
\end{aligned}
$$

where $s=\sum \alpha_{i}$ and $\alpha_{n+k}$ is defined to be $\alpha_{k}$ for all $k=1, \ldots, n-1$.

(ii) $\Rightarrow$ (iii) From the equation in (ii) it follows that

$$
x^{n}=x^{(r+1) n}
$$

and also

$$
\Pi x_{i}=\Pi x_{i}^{r m+1}
$$

for all integers $m \geqslant 0$. Therefore we have

$$
\begin{aligned}
\Pi x_{i} & =\Pi x_{i}^{r n+1}=x_{1}^{(r n+1)} \Pi_{i=2}^{n} x_{i}^{(r n+1)}=x_{1}^{(r(r+1) n+1)} \Pi_{i=2}^{n} x_{i}^{(r n+1)} \\
& =x_{1}^{\left(r^{2} n\right)} \Pi x_{i}^{(r n+1)}=x_{1}^{\left(r^{2} n\right)} \Pi x_{i} .
\end{aligned}
$$

(iii) $\Rightarrow$ (i) is trivial. 
REMARK. The equivalence of the conditions (i), (ii), and (iii) also follows from Theorem 7.11 of [5].

Note that the condition in (ii) means that in the subsemigroup $\mathbf{S}^{(n)}$ every element generates a finite cyclic group, and that the sizes of these groups are bounded (by $r$ ), which is equivalent to saying that $\mathbf{S}^{(n)}$ is the union of groups of bounded order. Also note that if $x_{1} \ldots x_{n}$ does not depend on one of its variables, the semigroup $S$ is $n$ nilpotent, and therefore the lemma above holds also for nonnegative integers $\alpha_{i}$. Hence, combining all the lemmas above we have

THEOREM 13. For a commutative semigroup $S$ and an integer $n \geqslant 2$ the following are equivalent:

(i) $\mathbf{S}$ is polynomially $(n-1)$-dense.

(ii) The term $x_{1} \ldots x_{n}$ is not uniquely representable in $\mathbf{S}$.

(iii) Either an equation $x_{1} \ldots x_{n}=x_{1}^{r+1} x_{2} \ldots x_{n}$ holds in $\mathbf{S}$ for some $r \geqslant 1$ or there is $a \in A$ such that the equation $x_{1} \ldots x_{n}=x_{1} \ldots x_{n} a$ holds in S.

(iv) The subsemigroup $S^{(n)}$ is either a monoid or the union of groups of bounded order.

In order to illustrate this theorem, we give some examples.

If a commutative semigroup $\mathbf{S}$ is $(n+1)$-nilpotent, but not $n$-nilpotent, it is polynomially $n$-dense, but not polynomially $(n-1)$-dense. This follows from the fact that $\mathbf{S}^{(n+1)}$ has only one element (and is therefore a monoid and a cyclic group), but the term $x_{1} \ldots x_{n}$ is uniquely representable (if it could also be written as another term, this term would have more factors, and would therefore be equal to a constant in $\mathbf{S}$, or it would have less variables, which shows that $x_{1} \ldots x_{n}$ could also be written as a term with more than $n$ factors).

The semigroup $\mathbf{N}_{\mathbf{0}}$ of all nonnegative integers with addition is a monoid and therefore it is polynomially 1-dense. On the other hand, the semigroup $\mathbf{N}$ of all positive integers with addition is not polynomially $n$-dense for any $n$. Indeed, it can be easily seen that $\mathrm{N}^{(n)}=(\{n, n+1, \ldots\},+)$, and this is neither a monoid nor a union of groups.

There exist also finite commutative semigroups which are not polynomially $n$-dense for any $n$. The smallest example we found is a four-element semigroup $S$ with 0 , given by: $a^{2}=a, b^{2}=b, b c=c b=c$, and $x y=0$ otherwise. Note that this implies that the lattice $E q c(S)$ is infinite; as a matter of fact, it is not difficult to see that a finite algebra $\mathbf{S}$ is polynomially $n$-dense for some $n$ if and only if the lattice $E q c(S)$ is finite. 


\section{REFERENCES}

[1] A. Day, 'Characterization of finite lattices that are bounded-homomorphic images or sublattices of free lattices', Canad. J. Math. 31 (1979), 69-78.

[2] G. Grätzer, Universal Algebra, (second ed.), (Springer-Verlag, Berlin, Heidelberg, New York, 1972).

[3] J. Ježek, N. Newrly and J. Tủma, 'Remarks on equational theories of semilattices with operators', Comment. Math. Univ. Carolin. 31 (1990), 417-425.

[4] J. Ježek, P. Pudlák, J. Tůma, 'On equational theories of semilattices with operators', Bull. Austral. Math. Soc. 42 (1990), 57-70.

[5] A. Kisielewicz, 'Varieties of commutative semigroups'. Preprint.

[6] N. Newrly, 'Equational density of clones of polynomial functions'. Preprint.

[7] D. Pigozzi and G. Tardos, 'The representation of certain abstract lattices as lattices of subvarieties' (to appear).

Institute of Mathematics

Technical University of Wroclaw

Wybrzeże Wyspiańskiego 27

50-370 Wrocław

Poland

kisiel@plwrtu11.bitnet
Technische Hochschule Darmstadt

Fachbereich 4 Schlossgartenstr. 7

6100 Darmstadt

Germany

xmatag01@ddathd21.bitnet 